\title{
Erratum
}

Monatsschr Kinderheilkd 2021 · 169:272-274 https://doi.org/10.1007/s00112-021-01123-x Online publiziert: 18. Februar 2021

(c) Springer Medizin Verlag GmbH, ein Teil von Springer Nature 2021
Die Online-Version des Originalartikels ist unter https://doi.org/10.1007/s00112-020-00881-4 zufinden.

Ernährungskommission der Österreichischen Gesellschaft für Kinder- und Jugendheilkunde (ÖGKJ) - Ernährungskommission der Deutschen Gesellschaft für Kinder- und Jugendmedizin (DGKJ) • Ernährungskommission der Schweizerischen Gesellschaft für Pädiatrie (SGP) · Deutsche Gesellschaft für Ernährungsmedizin (DGEM) · Nadja Haiden ${ }^{1}$

'Universitätsklinik für Klinische Pharmakologie, Medizinische Universität Wien, Wien, Österreich

\section{Erratum zu: Parenterale Ernährung von Früh-, Neugeborenen, Kindern und Jugendlichen}

\section{Konsensuspapier, basierend auf den Leitlinien der ESPGHAN, ESPEN, ESPR und CSPEN}

Erratum zu:

Monatsschr Kinderheilkd 2020

https://doi.org/10.1007/s00112-020-

00881-4

In • Tab. 4 (Zusammensetzung von für pädiatrische Patienten zugelassenen i.v.Lipidlösungen) kam es zu Übertragungsfehlern für die $a$-Tocopherolwerte bei den Fettemulsionen Sojabohnenöl/MCT-Öl und Sojabohnenöl/MCTÖl/Fischöl. In den • Tab. 6 (Empfohlene mittlere Zufuhr der einzelnen Vitamine bei Früh- und Neugeborenen) und - Tab. 15 (Empfohlene mittlere Tageszufuhr von Vitaminen in Abhängigkeit vom Lebensalter) sind die Einheiten für Vitamin B1 und Vitamin K nicht korrekt: Bei der empfohlenen Zufuhr für Vitamin B1 handelt es sich um Dosen im $\mu \mathrm{g}$-Bereich und nicht, wie in den Tabellen angegeben, um mg. Bei Vitamin K handelt es sich ebenfalls um Dosen im $\mu \mathrm{g}$-Bereich und nicht, wie angegeben, um I.E.

In - Tab. 15 ist zusätzlich die Einheit für Vitamin A nicht korrekt angegeben. Bei der empfohlenen Zufuhr handelt es sich um Dosen im $\mu$ g-Bereich und nicht, wie in der Tabelle angegeben, um I.E.

Des Weiteren sind in • Tab. 16 (Empfohlene mittlere Zufuhr von Spurenele-

\begin{tabular}{|c|c|c|c|c|c|}
\hline Inhaltsstoff & $\begin{array}{l}\text { Sojaboh- } \\
\text { nenöl }\end{array}$ & $\begin{array}{l}\text { Sojaboh- } \\
\text { nenöl/ } \\
\text { MCT-Öl }\end{array}$ & $\begin{array}{l}\text { Sojaboh- } \\
\text { nenöl/ } \\
\text { Olivenöl }\end{array}$ & $\begin{array}{l}\text { Sojaboh- } \\
\text { nenöl/ } \\
\text { Olivenöl/ } \\
\text { MCT-ÖI/ } \\
\text { Fischöl }\end{array}$ & $\begin{array}{l}\text { Sojaboh- } \\
\text { nenöl/ } \\
\text { MCT-ÖI/ } \\
\text { Fischöl }\end{array}$ \\
\hline Sojaöl (Anteil, \%) & 100 & 50 & 20 & 30 & 50 \\
\hline Olivenöl (Anteil, \%) & 0 & 0 & 80 & 25 & 0 \\
\hline $\begin{array}{l}\text { MCT (Kokosnuss, } \\
\text { Anteil, \%) }\end{array}$ & 0 & 50 & 0 & 30 & 40 \\
\hline Fischöl (Anteil, \%) & 0 & 0 & 0 & 15 & 10 \\
\hline a-Tocopherol (mg/l) & 21 & 132 & 32 & 164,5 & 177 \\
\hline Phytosterole (mg/l) & 423,1 & 268,5 & 266,1 & 170,6 & k. A. \\
\hline
\end{tabular}


Tab. 6 Empfohlene mittlere Zufuhr der einzelnen Vitamine bei Früh und Neugeborenen [2] Empfohlene Zufuhr pro kgKG und Tag

\begin{tabular}{|c|c|c|}
\hline Vitamin & Frühgeborene & Reifgeborene \\
\hline Vitamin A (I.E.) & $700-1500$ & $\begin{array}{l}500-1000 \\
\text { od. } 2300 / d\end{array}$ \\
\hline Vitamin D (I.E.) & $80-400$ & $40-150$ \\
\hline Vitamin E (I.E.) & $2,8-3,5$ & $2,8-3,5$ \\
\hline Vitamin $K(\mu g)$ & 10 & 10 \\
\hline Vitamin C (mg) & $15-25$ & $15-25$ \\
\hline Vitamin $B_{1}(\mu g)$ & $350-500$ & $350-500$ \\
\hline Vitamin $B_{2}(\mu \mathrm{g})$ & $150-200$ & $150-200$ \\
\hline Nikotinamid/Niacin (mg) & $4-6,8$ & $4-6,8$ \\
\hline Vitamin $B_{6}(\mu \mathrm{g})$ & $150-200$ & $150-200$ \\
\hline Panthotensäure (mg) & 2,5 & 2,5 \\
\hline Biotin $(\mu \mathrm{g})$ & $5-8$ & $5-8$ \\
\hline Folsäure $(\mu \mathrm{g})$ & 56 & 56 \\
\hline Vitamin $B_{12}(\mu \mathrm{g})$ & 0,3 & 0,3 \\
\hline
\end{tabular}

Tab. 15 Empfohlene mittlere Tageszufuhr von Vitaminen in Abhängigkeit vom Lebensalter [2]

\begin{tabular}{|lll}
\hline Vitamin & $\begin{array}{l}\text { Säuglinge bis 12 Monate } \\
\text { (pro kgKG und Tag) }\end{array}$ & $\begin{array}{l}\text { Kinder und Jugendliche } \\
\text { (pro Tag) }\end{array}$ \\
\hline Vitamin $A(\mu \mathrm{g})$ & $150-300$ & 150 \\
\hline Vitamin D $(\mathrm{I.E.})$ & $40-150$ & $400-600$ \\
\hline Vitamin E $(\mathrm{I.E.})$ & $2,8-3,5$ & 11 \\
\hline Vitamin $K(\mu \mathrm{g})$ & 10 & 200 \\
\hline Vitamin $C(\mathrm{mg})$ & $15-25$ & 80 \\
\hline Vitamin $B_{1}(\mu \mathrm{g})$ & $350-500$ & 1200 \\
\hline Vitamin $\mathrm{B}_{2}(\mu \mathrm{g})$ & $150-200$ & 1400 \\
\hline Nikotinamid/Niacin $(\mathrm{mg})$ & $4-6,8$ & 17 \\
\hline Vitamin $\mathrm{B}_{6}(\mu \mathrm{g})$ & $150-200$ & 1000 \\
\hline Panthotensäure $(\mathrm{mg})$ & 2,5 & 5 \\
\hline Biotin $(\mu \mathrm{g})$ & $5-8$ & 20 \\
\hline Folsäure $(\mu \mathrm{g})$ & 56 & 140 \\
\hline Vitamin $\mathrm{B}_{12}(\mu \mathrm{g})$ & 0,3 & 1
\end{tabular}

Tab. 16 Empfohlene mittlere Zufuhr an Spurenelementen [3]

\begin{tabular}{llllll} 
& \multicolumn{2}{l}{ Empfohlene Zufuhr pro kg KG und Tag } & Empfohlene Zufuhr pro Tag \\
\cline { 2 - 4 } Spurenelement & $\mathbf{0 - 3}$ Monate & $\mathbf{3 - 1 2}$ Monate & $\mathbf{1 - 1 8}$ Jahre & Maximum \\
\hline Zink & $250 \mu \mathrm{g}$ & $100 \mu \mathrm{g}$ & $50 \mu \mathrm{g}$ & $5 \mathrm{mg}$ \\
Kupfer & $20 \mu \mathrm{g}$ & $20 \mu \mathrm{g}$ & $20 \mu \mathrm{g}$ & $0,5 \mathrm{mg}$ \\
Selen & $2-3 \mu \mathrm{g}$ & $2-3 \mu \mathrm{g}$ & $2-3 \mu \mathrm{g}$ & $100 \mu \mathrm{g}$ \\
Chrom & - & - & - & $5 \mu \mathrm{g}$ \\
Mangan & $\leq 1 \mu \mathrm{g}$ & $\leq 1 \mu \mathrm{g}$ & $\leq 1 \mu \mathrm{g}$ & $50 \mu \mathrm{g}$ \\
Molybdän & $0,25 \mu \mathrm{g}$ & $0,25 \mu \mathrm{g}$ & $0,25 \mu \mathrm{g}$ & $5 \mu \mathrm{g}$ \\
lod & $1 \mu \mathrm{g}$ & $1 \mu \mathrm{g}$ & $1 \mu \mathrm{g}$ & - \\
Eisen & $50-100 \mu \mathrm{g}$ & $50-100 \mu \mathrm{g}$ & $50-100 \mu \mathrm{g}$ & $5 \mathrm{mg}$
\end{tabular}

menten) die Überschriften der Altersgruppen verrutscht. Irrtümlich wurde die Altersgruppe 3-12 Monate zweimal angegeben statt 3-12 Monate, 1-18 Jahre und Maximum. Dies wurde korrigiert.

Anbei die korrekten Tabellen mit entsprechenden Werten.

Wir bitten die Fehler zu entschuldigen und die korrekten Angaben zu berücksichtigen.

\section{Korrespondenzadresse}

Assoz. Prof. PD Dr. Nadja Haiden, MSc.

Universitätsklinik für Klinische Pharmakologie, Medizinische Universität Wien

Währinger Gürtel 18-20, 1090 Wien, Österreich nadja.haiden@meduniwien.ac.at

\section{Literatur}

1. Cai W et al (2018) Biological and Clinical Aspects of an Olive Oil-Based Lipid Emulsion-A Review. Nutrients. 10(6):776. https://doi.org/10.3390/ nu10060776

2. Bronsky J, Campoy C, Braegger C, nutrition EEECwgopp (2018) ESPGHAN/ESPEN/ESPR/CSPEN guidelines on pediatric parenteral nutrition: Vitamins. Clin Nutr 37(6 Pt B):2366-2378. https:// doi.org/10.1016/j.cl.nu.2018.06.951

3. Domellöf M, Szitanyi P, Simchowitz V, Franz A, MimouniF, nutritionEEECwgopp (2018)ESPGHAN/ ESPEN/ESPR/CSPEN guidelines on pediatric parenteral nutrition: Iron and trace minerals. Clin Nutr 37(6 Pt B):2354-2359. https://doi.org/10. 1016/j.cl.nu.2018.06.949 
Hier steht eine Anzeige.

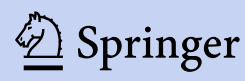

